\title{
Estrategias metodológicas para la enseñanza-aprendizaje con enfoque aprender en libertad
}

\section{Methodological strategies for teaching-learning with focus learning in freedom}

\author{
Mayra Cecilia Cruz Mangui \\ Universidad Internacional del Ecuador, Ecuador \\ Milton Alfonso Criollo Turusina \\ Instituto Ecuatoriano de Seguridad Social, Ecuador \\ Deida María de Fátima Raffo Velarde \\ Universidad de Guayaquil, Ecuador \\ Autor para correspondencia: maycruzma@uide.edu.ec, dancercriollo@gmail.com, \\ deida.raffov@ug.edu.ec \\ Fecha de recepción: 28 de Agosto de 2017 - Fecha de aceptación: 30 de Septiembre de 2017
}

Resumen: Son muchas las estrategias educativas que tiene a su alcance el docente a la hora de impartir los conocimientos al alumnado, tomando por base la actitud vanguardista de los estudiantes a la hora de elegir el campo de estudio, se concede un valor agregado a la libertad de buscar nuevos conocimientos a través de un campo ligero, con medios agradables para los educandos y que manifieste el interés común del grupo de estudio. Dentro del campo investigativo la asignatura de lenguaje integral, proporciona al alumno las herramientas necesarias para desarrollar la capacidad de estructurar correctamente todas las manifestaciones del lenguaje hablado y escrito. La música por su parte, utilizada como recurso didáctico va a estimular el aprendizaje significativo, ya que en el medio actual los jóvenes y adultos se encuentran altamente motivados por la misma; por lo que toda idea de adquirir conocimiento dentro de un área específica apoyándose en otra, se transforma en una innovación pedagógica y andragógica, lo cual contribuye significativamente al campo heurístico de la investigación. Consecuentemente, el área musical fue elegida para complementar el interés por escribir y expresarse correctamente en el alumnado, expresando así de manera libre su pensamiento crítico dentro de una estructura musical, el cual se encuentra presente en la letra que se elija, la misma que va a variar dependiendo del género musical dramático, eufórico, simple o de gran estructura gramatical.

Palabras Clave: estrategia metodológicas; música; lenguaje integral

Abstrac: There are many strategies which the educators have when they share their knowledge with their students, it is based on students 'vanguard attitude when they make decision to study a specific area, it gives an added value to look for new knowledge through a light field, and the pupils are interested in common work through comfortable resources. Integral language issue investigation provides fulfill tools to students in order to develop the capability of structuring all expressions of written and spoken language correctly. Other hand, music, it is used as didactic resource, is going to encourage the meaning learning because the our current environs, young and old persons are highly motivated because of it; thus, the idea of adquiring knowledge in a 
specific area based on others, it transforms inot a pedagogical and andragogic innovation which it meaningfully helps heuristic investigation method. As a result, musical area was selected in order to complete in students interests in writing and speaking correctly, so they can precise their critical thinking about music related to lyrics which they can contrast according to dramatic style, simple, euphoric , or challeging gramatical structure.

Key words: methodological; music; integral language

\section{Introducción}

Toda expresión es comunicación, para comunicar debemos interactuar, y la mejor forma de interactuar es por el método de creación colectiva propuesta por Enrique Buenaventura, que consiste en cohesionar interpersonalmente al alumnado, para consolidar en materia las diferentes ideas que de su imaginación emana.

De tal forma que el alumnado consolidado con sus relaciones interpersonales por sus diferentes modelos paradigmáticos, se motiven con el fin de adquirir nuevos conocimientos estimulados por el campo de la música, lo cual culminará con el desarrollo de capacidades para estructuraciones y reestructuraciones del lenguaje, oral, escrito y corporal en su máximo esplendor, y la transformación de un individuo con la capacidad de expresarse correctamente.

(Buenaventura, 2009) El método es la configuración que adopta el proceso docente educativo en correspondencia con la participación de los sujetos que en él intervienen, de tal manera que se constituye en los pasos que desarrolla el sujeto, en su interacción con el objeto, a lo largo de su proceso consciente de aprendizaje.

La forma explícita de cómo nos damos a entender o emitimos nuestros pensamientos se llama lenguaje, para su ideal funcionamiento en el ser humano, necesita de una serie de normas dialécticas y ortográficas. Esto sin duda ocasiona poco interés en varios individuos de diferentes instituciones educativas, ya que les parece monótono o un tanto aburrido la forma de como adquieren dicho conocimiento. Sin embargo, el poco interés de las personas en aprender a escribir o a hablar correctamente no los inhibe de su responsabilidad para con el desarrollo de una sociedad.

Por otro lado, los docentes tienen la responsabilidad de contribuir en la dinámica del proceso de enseñanza aprendizaje, con nuevas estrategias metodológicas que se estructuren y se acoplen a las vanguardistas tendencias en los modelos paradigmáticos que los jóvenes adquieren, rompiendo el lazo de la concepción que poseen sobre la educación, que obliga a hacer algo, sin demostrar estimulo diferente que el aprobar o sacar una buena nota en una determinada asignatura.

Para (Bocaz Claudia Silva, Rocío campos, 2003) en su investigación sobre el método María Montessori, un individuo motivado por una curiosidad natural continuará aprendiendo luego de horas o días, de haber concluido su proceso educativo dentro del salón de clases. Esta concepción coincide en la meta de la presente propuesta, que es cultivar el deseo natural por aprender, brindando una nueva metodología de enseñanza aprendizaje con el instinto, la naturalidad, y la autonomía que da la educación en libertad.

El sistema educacional ecuatoriano, tiene ya completo la transitoriedad del conductismo hacia el constructivismo, el mismo que busca brindar un proceso dinámico a la aprehensión del 
conocimiento, partiendo de otros previos propios del individuo, bases sostenibles en la teoría de Piaget.

Mediante el análisis de las letras de canciones preferidas por los alumnos, se emitirá un juicio crítico, de las mismas, para razonar de acuerdo a su estructura gramatical, la correcta escritura y optimización de su expresión lingüística en las canciones simples, o para tomar de referencia como modelo gramatical, las letras de canciones de gran estructura.

\section{Connotaciones motivacionales del proceso aprender en libertad}

La conducta del ser humano es la acción o resultado de la determinación de sus actos ocasionados por un estímulo, a este último lo llamaremos motivación. Aprender en libertad consiste activar o modificar en aumento la conducta del alumnado, con relación a la adquisición de conocimientos. Este proceso debe reflejar tres componentes:

- Elemento de valor

- Elemento de expectativa

- Elemento Afectivo

(Núñez, 2009)

\section{El Elemento de valor}

Hace referencia a la causa de realización de una tarea en una asignatura, determinada por la valoración de primacía o escasa importancia en la realización del proceso de aprendizaje. En este elemento el educando se preguntará: ¿Por qué realizaré dicha tarea?

\section{Elemento de expectativa}

Interactúa con las relaciones intrapersonales del educando, pues hace referencia a la apreciación de efectividad que posee el mismo, para considerarse o no autosuficiente en el contexto académico. En este elemento el educando se preguntará: ¿Estoy capacitado para realizar o no, aspectos académicos?

\section{Elemento Afectivo}

Tiene como fin el determinar cualitativamente el nivel de emociones, reacciones y sentimientos como resultado de la realización del proceso de aprendizaje. En este elemento el sujeto se preguntará ¿Cómo me siento al realizar una tarea?

Calificando los elementos motivacionales tendremos como consecuencia lo dificultoso que será para el alumno mostrarse estimulado al efectuar trabajos o tareas universitarias cuando se sientan con incapacidad de realizarlo (elemento de expectativa), si la actividad a desempeñar no posee ningún interés hacia él (elemento de valor), o si le resulta fastidioso, cansando, aburrido o desagradable (elemento afectivo). Sin embargo, equilibrando correctamente los tres elementos, lograremos implicar al educando en un proceso con juicio de autoeficacia, productividad, valor en el rendimiento y funcionalidad. Todo esto causando un cambio significativo en la conducta motivacional hacia el proceso académico.

La motivación es un factor indispensable al momento de consolidar el proceso de enseñanza aprendizaje en el educando. En este aspecto, la música será utilizada como elemento 
motivacional en dicho proceso, no obstante, al hablar de música no necesariamente el educador debe ser un intérprete o experto de la misma, ya que en este campo no educaremos al alumno en materia de entonación ni estudio de un determinado instrumento musical; más bien buscaremos despertar en el educando el interés por adquirir un conocimiento lingüístico, pues más allá de la melodía, armonía y ritmo que posee la misma, también se encuentra acompañada por letras de gran estructura gramatical, con cantantes de timbre atractivo, o encontraremos varios poemas que con el paso del tiempo han sido añadidos a un ensamble musical o viceversa.

\section{El método de creación colectiva en la motivación}

El desarrollo de la motivación en el individuo, no debe finalizar en conductas de superioridad hacia sus compañeros, ni distorsionar la motivación dirigiéndola hacia una tendencia para evitar el fracaso, o juicios negativos sobre su capacidad cognitiva. Para evitar esto, el método de creación colectiva proporciona un direccionamiento apropiado en el afianzamiento de las relaciones interpersonales.

\section{Impacto musical}

Está comprobado que la música influye en el estado anímico del ser humano, capaz de llevarlo a los extremos de una intensa alegría a la depresión extrema. Las emociones pueden ser controladas con la música, de hecho, se piensa que las personas buscan acompañarse de la música por su temor a estar solas con ellos mismos, es una forma de negación al pensamiento.

Como expresa (Educarm, La Filosofía de Platón, 2010) "...mencionaremos algo importante para la ética y la política de Platón: la teoría de las tres almas, o mejor, de las tres "partes" (el alma es inmaterial y simple, no tiene partes) o facultades del alma: racional, impulsiva y pasional." Las emociones son afectadas por la acumulación de experiencias personales e intelectuales, lo cual conlleva a la llamada "madurez emocional"; sin embargo, el proceso de crecimiento personal es individual y disímil para cada ser humano por los factores heterogéneos que pueden incidir en el mismo.

Es entonces Platón con su teoría del conocimiento donde afirma que el objetivo del mismo, es encontrar una definición para todo lo que nos rodea. Entonces, nos preguntamos... ¿por qué los cambios de conducta al escuchar una música determinada? el dolor, la euforia, el desenfreno, tiene su fundamento en la memoria a corto y largo plazo, de hecho, una melodía puede traer a la mente todo un episodio del pasado, pudiendo incidir en el estado anímico del momento en tiempo real. Platón contaba con habilidades de orador, que le permitió influir en su época de manera tal que hasta la actualidad se lo cita:

(clásicos, 2015) Su desentendimiento de las ideas morales fue mucho más claro y resuelto que el de Protágoras; su enseñanza tenía por exclusivo objeto el arte de triunfar en la vida pública, sin empleo de violencia exterior, por la fuerza mágica de la oratoria. Este arte contaba ciertamente con la eficacia del razonamiento que domina la inteligencia, pero mayormente con el hechizo ejercido en el alma por el elemento sensible, la música de la lengua. Del éxito oratorio se derivan el honor, la gloria y el poder, que es todo cuanto puede ambicionarse; el fin inmediato del discurso, el sentido en que ha de mover los ánimos es indiferente. 
Por ello, este estudio considera que la aplicación libre de la música y el efecto significativo de la letra puede ser utilizado como estrategia de enseñanza para el desarrollo de la inteligencia emocional, ya que, al realizar estudios sobre las competencias y habilidades del ser humano, como el ímpetu, autocontrol, motivación; son cualidades asimilables que pueden desarrollarse y superarse en el transcurso de los años y la asimilación de experiencias positivas y negativas.

El análisis de la inteligencia emocional la hace Howard Gardner, que toma como referente la música clásica, que, por su armonía, influye en las emociones de manera directa, con lo cual el receptor va a ser capaz de determinarlas y dirigirlas. Tomando en consideración lo expuesto por Gardner, se considera posible el discernimiento de las emociones personales y con ello la capacidad de conducirlas de manera correcta.

\section{Avance curricular a partir de la creación musical}

En esta etapa se indica que la razón de ser de este artículo no es enseñar al alumno a componer canciones, sino ubicarlos dentro de su entorno para que acorde con sus preferencias musicales pueda transmitir sus experiencias y convertirlas en saberes, contando con el apoyo e incentivo metodológico del profesor.

Acorde a lo expresado por Montserrat Sanuy (Swanwick, 2000) Tan sólo tres palabras Música, Pensamiento, Educación- han servido a SWANWICK para sintetizar el contenido esencial de su obra y estimular la atención de un buen lector. El autor plantea oportunamente el hipotético conflicto entre la teoría y la práctica, entre el pensamiento abstracto y la realización concreta, entre el mundo teórico y el marco de lo operativo. A este propósito escribe que "hacer las cosas sin la gula del pensamiento puede ser inútil y hasta peligroso". Merece subrayarse el mérito de su valoración de lo teórico dentro de un mundo tal vez excesivamente dominado por la praxis.

Encontramos entonces la convergencia de la corriente educativa en libertad, utilizando la música como estrategia metodológica, el pensamiento sobre el que se va a trabajar para su transformación y la educación mediante la cual se desarrolla el currículo. De este formato tripartito surge el planteamiento de un Diseño Curricular con enfoque holístico, basado en la necesidad de comunicación que tiene el ser humano, aprovechando la riqueza de nuestra lengua en sus formas literaria y musical.

Los niveles educativos en base a programas de la asignatura de música persisten en las mallas curriculares: (Swanwick, Music, Mind and Education, 2000)

\begin{tabular}{|c|c|}
\hline Nivel físico & Nivel psicológico \\
\hline - Frecuencia & tono \\
\hline - Intensidad & altura \\
\hline - Forma & timbre \\
\hline - Duración & tiempo \\
\hline
\end{tabular}

Dejando de lado el carácter funcional del mismo, dejando oculto la apreciación libre de la música, en los experimentos tratados se evidencia claros conceptos opuestos sobre la misma melodía, acorde a los conceptos del autor se indicaba: 
(Swanwick, Music, Mind and Education, 2000) No se produjo coincidencia, cosa nada sorprendente porque el material sonoro aislado tiende a ser lo que cada individuo decide hacer de él. Por ejemplo, en uno de estos experimentos 11 personas calificaban la tercera menor de "triste" o "dolorida", frente a 26 que calificaban ese carácter a la tercera mayor. Los materiales musicales son claramente ambiguos. Dentro de la tonalidad occidental una tercera menor se puede escuchar como la mitad superior de una mitad superior incompleta, y una tercera mayor como parte de un acorde menor. Incluso a este nivel tan limitado de experiencia musical los oyentes aportan sus propias expectativas.

La propuesta curricular que plantea Swanwick, intenta romper paradigmas educativos pasando de lo teórico a lo práctico, sin descuidar el nivel académico, con la finalidad que el alumno asimile las asignaturas correspondientes a su malla curricular ayudado por los sonidos musicales de su predilección. El perfil del docente del nuevo siglo debe ser innovador, considerando que la metodología conductista aún se encuentra firmemente establecida en algunos de los medios educativos con mucha resistencia a desaparecer, pero afortunadamente las nuevas corrientes educativas van evolucionando dentro de la academia, impulsando al docente a la actualización de sus conocimientos y en muchos casos lo lleva a reaprender metodológicamente los procesos de enseñanza aprendizaje.

\section{Sistema lingüístico}

La aplicación de este modelo pedagógico, con énfasis en la libertad, no nos aparta del rigor científico de la lingüística aplicada, ya que esta rama es interdisciplinaria. Algunos psicólogos como Chomsky aportan con sus estudios investigativos sobre los principios de adquisición del lenguaje:

El psicólogo educativo español (Regade, 2012) explica la teoría de lenguaje de Chomsky, el lenguaje humano nos permite expresar infinidad de ideas, informaciones y emociones. En consecuencia, el lenguaje es una construcción social que no para de evolucionar. La sociedad va marcando las pautas sobre las normas y usos comunes del lenguaje, tanto en su versión oral como en la escrita. De hecho, es muy común que los niños empleen el lenguaje de forma muy particular: mezclando conceptos, inventando palabras, deformando otras, construyendo las frases a su manera... Poco a poco, su cerebro va asimilando las reglas y recurrencias del idioma, cometiendo cada vez menos errores y usando con propiedad el amplio abanico de artefactos que les brinda el lenguaje.

La lingüística estructurada que de manera sistémica en la que (Soler, 2002) "incluye la semántica como componente interpretativo de la estructura profunda (...) y en segundo lugar cabría señalar la influencia que han ejercido diversas disciplinas en la configuración de la lingüística textual."

Además Soler da un paseo por las fuentes teóricas de los procesos de aprendizaje, resultando como fuente enriquecedora de conocimientos pedagógicos, como los que expresa en su texto sobre metodología de enseñanza:

(Soler, 2002) Desde la óptica de la teoría del aprendizaje, hay que señalar que, al igual que la teoría del innatismo y la teoría del lenguaje de Chomsky, se apuesta por un enfoque mentalista 
y cognitivo. Las teorías cognitivas de Piaget (1929) y Ausubel (1968), entre otros, y la teoría socio-cognitiva de Vygotsky (1977), ejercerán una notable influencia en la didáctica de las lenguas. Piaget (1929) y Ausubel (1968) partiendo, de la teoría constructivista, consideran el aprendizaje del lenguaje como una representación. Ahora bien, mientras Piaget sostiene que el lenguaje se construye, al igual que cualquier comportamiento perceptivo, en el marco de relaciones que se establecen con otros seres humanos y el entorno exterior, Ausubel enfatiza la idea de que el aprendizaje es un proceso activo de construcción de esquemas a partir de información significativa. Vygotsky, por su parte, insiste más en la naturaleza del lenguaje como instrumento de intercambio social.

Considerando los aportes teóricos citados, el sentido nominal de los aspectos comunicativos que involucran al lenguaje depende de los factores que intervengan en la emisión del mismo, valorando las habilidades y competencias que se interpongan en la facultad cognitiva del estudiante.

\section{La libertad de crear}

Considerando que la capacidad de crear no puede estar cuadrada por limitaciones mentales, se le confiere al alumno la capacidad de proponer ideas propias para la ejecución de los trabajos educativos, aportando ideas propias para la interiorización del estudio. En consecuencia, si la música se va a utilizar como una herramienta de comunicación para la enseñanza aprendizaje, se deberá trabajar en la correcta aplicación de la música con el fin de expresarse.

(Ministerio de Educación, 2001) El lenguaje verbal se utiliza para dialogar, exteriorizar ideas y sentimientos, comunicarse con otros; el escrito, para plasmar ideas. En la música, hablar equivaldría a improvisar y escribir (en el sentido de redactar o crear), a componer, aunque la composición no siempre deba ser registrada mediante los signos de la notación musical. Cada uno de estos dos tipos de actividades, improvisación y composición, presenta características propias y requiere de la utilización de diferentes técnicas y recursos.

Aunque la creatividad nace espontáneamente en el individuo, pueden aparecer aspectos positivos y negativos, que se evidencian en los estereotipos seguidos donde el alumno no acepta pensar sino seguir, por resultarle más cómodo y menos laborioso. A la inversa, se puede valorar la capacidad ilimitada de propuestas estudiantiles, donde aparentemente la puerta que se encontraba cerrada se abre de par en par, dejando entrar todo un torrente de ideas valoradas en aspectos significativos para el aprendizaje propio, pero también se lo puede tomar como un aporte metodológico de naturaleza empírica.

\section{Análisis cualitativo del efecto musical}

En el Ecuador no se ha realizado aún un estudio valorativo de la influencia de la música como herramienta pedagógica de aprendizaje, sin embargo, en España encontramos una investigación sobre la educación musical aplicada a ese contexto educativo.

(García, 2000) La investigación cualitativa pretende dejar claro ante todo que las complejas relaciones humanas no pueden ser tratadas exclusivamente con frías ecuaciones matemáticas que olvidan tanto el contexto en el que se desarrollan dichas relaciones como las interpretaciones y significados que éstas adquieren para los sujetos que las llevan a cabo. Como escribe Savater, "los 
humanos no somos problemas o ecuaciones, sino historias; nos parecemos menos a las cuentas que a los cuentos" (SAVATER, 1997:139). En el enfoque cualitativo no se concibe silenciar la opinión de los objetos estudiados puesto que básicamente se trata de seres humanos; antes al contrario, esta opinión es objeto de análisis, reflexión y contraste crítico por parte de los diferentes participantes en el proceso de investigación para, entre todos, lograr encontrar las claves de los comportamientos que caracterizan un determinado evento social. Como escribe Torres, "nuestras acciones están condicionadas por los significados que otorgamos a las acciones de las personas y a los objetos con los que nos relacionamos. Una investigación que descuide estos aspectos está claro que no reflejará todas las dimensiones de esta realidad, e incluso podríamos decir que captará lo menos revelador de ella" (TORRES, 1988:13).

Rodríguez y García aportan con un elemento fundamental a la investigación; consideran a la educación como un proceso y a la enseñanza musical como copartícipe de la educación integral del ser humano. Los aspectos cualitativos de estudio se van a enfocar en la intencionalidad de realizar propuestas que aporten al mejoramiento del sistema educativo con aspectos creativos y su desarrollo significativo en el eje curricular.

\section{Determinación de la música en la juventud}

En la actualidad la música marca tendencias y la libertad para elegirla va a depender del gusto, circunstancias, medio social y económico, entre otras causas que influyen en la decisión o preferencia de los jóvenes, y se toman algunos indicadores básicos que los determinan según (Ortí, 2013):

1- Aspectos Demográficos

2- Aspectos Socioeconómicos

3- Convivencia

4- Valores

5- Uso De Las Tics

6- Ocio Y Tiempo Libre

7- Drogas

8- Participación

9- Las Relaciones (Grupales, Sexuales)

10- Aspectos Culturales

11- Tipologías

12- Indicadores Opináticos sobre la Crisis

13- La Juventud vista desde los Adultos

14- La Juventud en los Medios

Tomado de los estudios de Anna Sanmartín, la autora señala e14 indicadores básicos de estudio de los jóvenes, en una sociedad del primer mundo como es la española. Circunstancialmente, van a cambiar de acuerdo a su medio, pero de manera intrínseca se puede anotar que en todos los aspectos se relaciona juventud con la música. Los parámetros estadísticos se reflejan en el estudio realizado a nivel de problemas de conducta juveniles, específicamente la drogadicción, sin embargo, sirve de análisis comparativo para contraponerlo con el objeto del presente artículo que se trata del aprendizaje en libertad. 
Pero es interesante evaluar, para esto se deben realizar una serie de cuestionamientos que formularían una respuesta adecuada, por ejemplo:

$\checkmark \quad$ ¿Cuál es el tipo de música que los jóvenes prefieren en la actualidad?

$\checkmark \quad$ ¿Cuáles son las fuentes de información que cumplen con sus requerimientos?

$\checkmark \quad$ ¿Siguen alguna influencia familiar para su preferencia musical?

$\checkmark \quad$ ¿Qué tipo de equipos utilizan para escuchar música?

En un estudio en la ciudad de Lima, Perú, se evidenció un fenómeno musical de retroceso. (Arana, 2015) 54,3\% de los jóvenes prefiere la música de los años 80. En Lima y el Callao. En cambio 39,7\% de encuestados, con una edad entre 18 y 29 años, prefiere las creaciones musicales de la actual década, según reciente sondeo del Instituto de Opinión Pública de la Universidad Católica. Una importante mayoría de ese sector considera que las composiciones de décadas pasadas son de mayor calidad. Una mayoría accede a la música a través de la radio.

Realiza un análisis de la preferencia musical en los jóvenes, y salta a la vista los resultados que no son para nada los que se esperaba. Esta aparente contradicción lleva a un nuevo análisis, en donde la década del 80 marca un referente para las generaciones subsiguientes y se manifiesta en una comunicación entre generaciones que han crecido escuchando la música de aquella época. Las TIC's empleadas como buscador también se las emplea como herramienta inmediata en el ambiente musical y las aplicaciones como youtube nos conectan en red con cualquier manifestación del pasado.

Pero algo más importante de anotar es lo que indica (Arana, 2015): Según Eleazar Valverde Chale, figura de Radio Filarmonía, la explicación de esta preferencia va por otra dirección: la falta de calidad de las creaciones actuales. Señala que las canciones de décadas pasadas poseían mucho cuidado en la elaboración de la letra y la melodía, características que las convierten en 'clásicos' y, por lo tanto, siempre vigentes a través de varias generaciones. "Aunque también la palabra de los padres es la que manda, al decirles a sus hijos que la música de antes era mejor y esto ha influenciado en la decisión de los chicos (ríe)", añade. Este punto se refuerza con el 72,8\% de jóvenes (entre 18 y 29 años) que consideran que las composiciones del pasado son superiores en cuanto a calidad respecto a las del presente. En cambio, solo el 22,5\% de jóvenes cree lo contrario.

En este estudio de casos se encuentran un número de jóvenes que han heredado los gustos de sus padres porque fue lo que tuvieron a su alcance, o porque de tanto escucharlo lo rememoran como parte de su infancia. No obstante, un número considerable de jóvenes construyen su acervo musical en el transcurso de su vida y lo acumula en toda la variedad que se refleja.

Según Ana y Oscar Bressan, en una muestra de un grupo poblacional podríamos conocer los gustos musicales en proporciones de jóvenes encuestados, sin embargo, no tendríamos un valor real a menos que lo repitamos en cada uno de los entornos sociales que los determinan.

(Bressan \& Bressan, 2008) Análogamente, si quisiéramos estudiar los gustos musicales de los chicos de una localidad, nuevamente deberíamos tratar de encontrar una muestra atendiendo a las distintas edades (sus gustos van evolucionando con la edad), a los distintos barrios, a los distintos sectores sociales, etcétera. 
En la era tecnológica actual, no significa ningún problema la portabilidad musical, por tanto, puede llegar a cualquier parte y con la gama de variaciones que existen. Los sonidos del siglo XX hasta la actualidad han avanzado sin filtro, en consecuencia el uso de los aparatos tecnológicos como es ilimitado, según los estudios de Bull, esto es lo que opinan los jóvenes sobre la forma de escuchar música mediante un Ipod (Bull, 2010):

- «He llegado a la conclusión de que el hecho de que la música se pueda llevar de un sitio a otro es esencial en mi vida. Me llevo el iPod cuando voy a darme un baño relajante. Si mi compañera está viendo la televisión lo escucho mientras hago la cena. Lo uso cuando me duermo» (Ben).

- «Ahora escucho música cuando puedo. Al ir o volver del trabajo, en el trabajo, de vacaciones, en el tren o en el avión, incluso en casa cuando no quiero molestar a mi compañero. Cualquier canción que quie ro escuchar está al alcance de mis manos en cualquier momento» (Susan).

- «Entonces empecé a llevarlo -el iPod-cuando iba a la compra. Lo hacía para controlar mi entorno y hacerme insensible a todo lo que estuviera a mi alrededor. Lo que me pareció interesante fue que cuanto más llevaba el iPod menos quería relacionarme con desconocidos. He llegado al punto en que no establezco contacto visual. Me siento como encerrada en una burbuja... La gente se convierte más en una elección cuando llevo el iPod» (Zuni).

- «Me gusta escuchar sin que me interrumpan, y si me interrumpen me siento un poco como si me asaltaran. Escucho el iPod cuando estoy solo. Es mi tiempo y mi espacio, y cualquier interrupción invade el tiempo que dedico a mí mismo» (Matt).

- «No quiero que nadie me moleste cuando estoy escuchando. No me interesa hacer nada excepto es cuchar música cuando tengo los auriculares puestos» (Wes).

Alicia Miranda Díaz, hace un análisis estadístico de (Díaz, 2015)

\section{1. ¿En qué momentos del día escuchas música?}

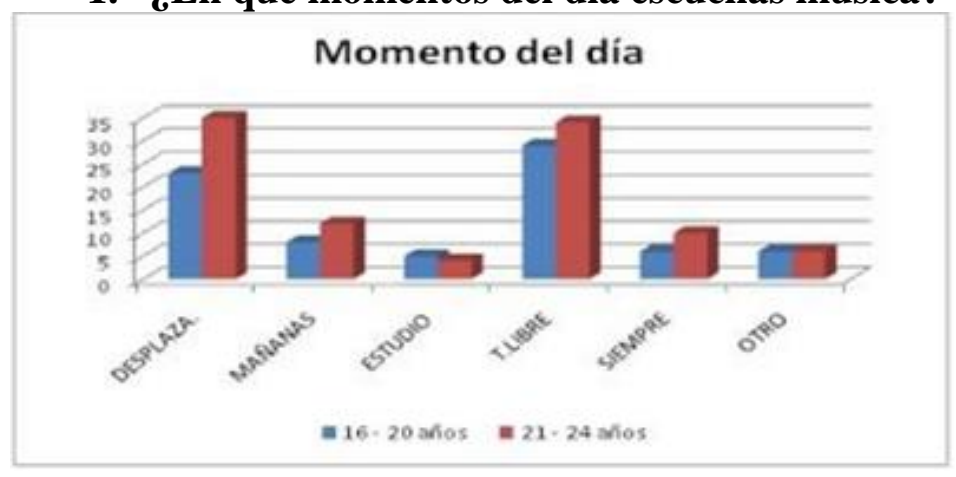

Un $68,2 \%$ del total de los encuestados escucha música durante los desplazamientos, un $23,5 \%$ lo escucha por las mañanas, un 10,5\% durante el estudio, el 74,1\% durante el tiempo libre, el $18,8 \%$ a todas horas y el $14,1 \%$ en otro momento.

A continuación, se realizará una tabla comparativa, con los resultados obtenidos entre los dos grupos de encuestados, entre los que están las personas de edades comprendidas entre 16 y 20 años y los de 21 y 24 años. 


\begin{tabular}{lrl}
\hline Lugares & $\mathbf{1 6 - 2 0}$ & $\mathbf{2 1 - 2 4}$ \\
\hline Desplazamientos & $48,90 \%$ & $92,10 \%$ \\
Mañanas & $17 \%$ & $31,50 \%$ \\
Estudio & $10,60 \%$ & $10,50 \%$ \\
Tiempo libre & $61,70 \%$ & $89,40 \%$ \\
A todas horas & $12,70 \%$ & $26,30 \%$ \\
Otro momento & $12,70 \%$ & $15,70 \%$ \\
\hline
\end{tabular}

Con los resultados obtenidos se puede determinar que, por lo general la gente escucha más música durante su tiempo libre que en el resto de los momentos del día, siendo destacable este dato en ambos grupos de edad. Otra pregunta destacable dentro del estudio de Alicia Miranda (Díaz, 2015)

\section{7. ¿Qué estilo de música escuchas?}

Esta es una de las preguntas que más variedad de resultados ha tenido, ya que los gustos de estilos musicales pueden ser tantos como personas en el mundo. En este caso, debemos tener en cuenta que los jóvenes entrevistados pertenecen a un mismo grupo cultural, y se encuentran dentro del mismo contexto social, de ahí sus similitudes.

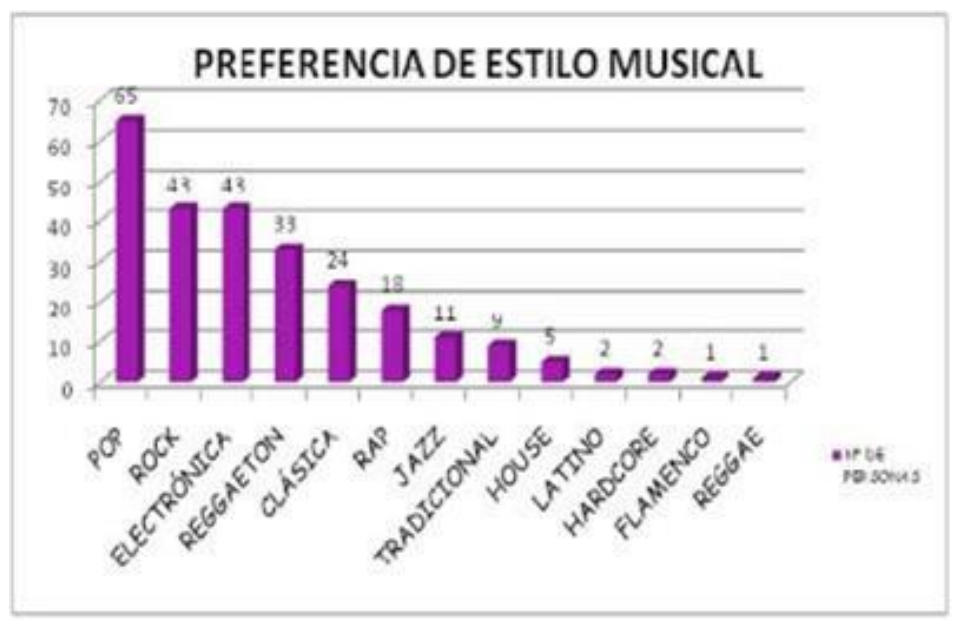

Para verlo de un modo más claro, expondremos otro gráfico en el que cada etiqueta es el tanto por ciento con respecto al total de los encuestados. De este modo, vemos que un 76,47\% de los encuestados ha elegido el pop como uno de los géneros de su agrado. Es el género más escuchado por las personas de la muestra, y al mismo tiempo el más popular en nuestro país. Por otro lado, podemos ver que más de la mitad de los sujetos ha afirmado que escucha rock y música electrónica, ambos con una aceptación de 50,59\% sobre el total de encuestados.

En el caso opuesto están géneros como el flamenco, el reggae, el hardcore o la música latina, con menos de un 3\%. Además, estos géneros ni siquiera aparecían en la lista inicial de respuestas, sino que fueron añadidos por los encuestados. 


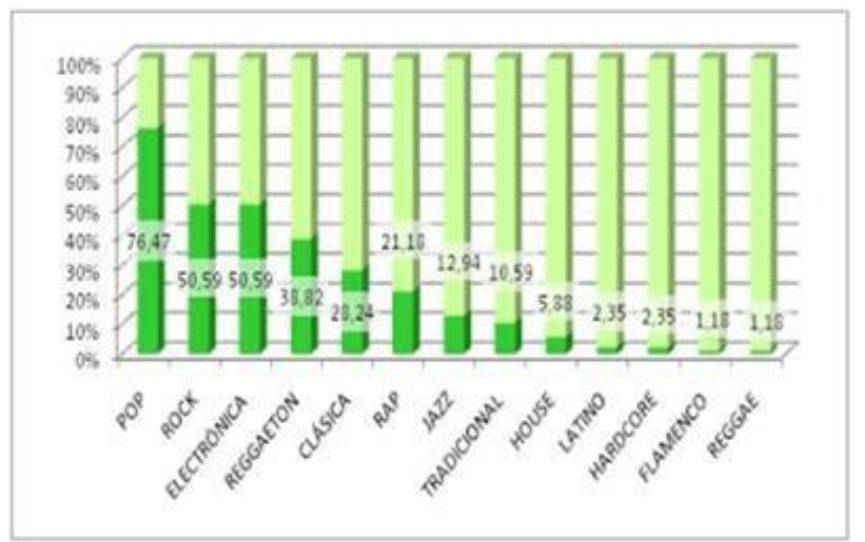

En cuanto al tiempo que los jóvenes dedican a escuchar música, la misma autora ha plasmado en gráficos por rango de edad, vamos a tomar la edad en jóvenes entre 16 y 24 años (Díaz, 2015):

\section{0. ¿Cuánto tiempo dedicas a escuchar música diariamente?}

\section{6-20 años}

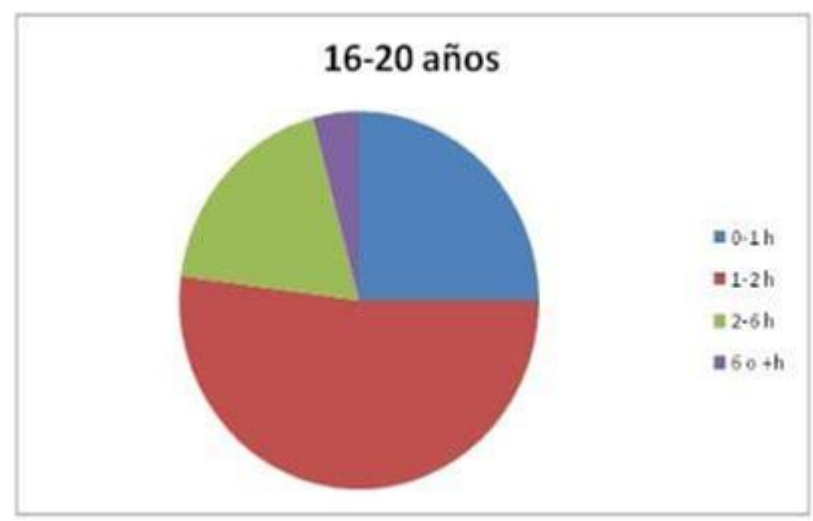

Como podemos observar en el gráfico, más de la mitad de las persona encuestadas de 16 a 20 años escuchan música entre 1 y 2 horas al día. En torno al resto de los encuestados, la opción más escogida es $0-1$ horas, seguido de 2 a 6 horas, y finalmente, un pequeño tanto por ciento escucha música más de 6 horas.

\section{1-24 años}

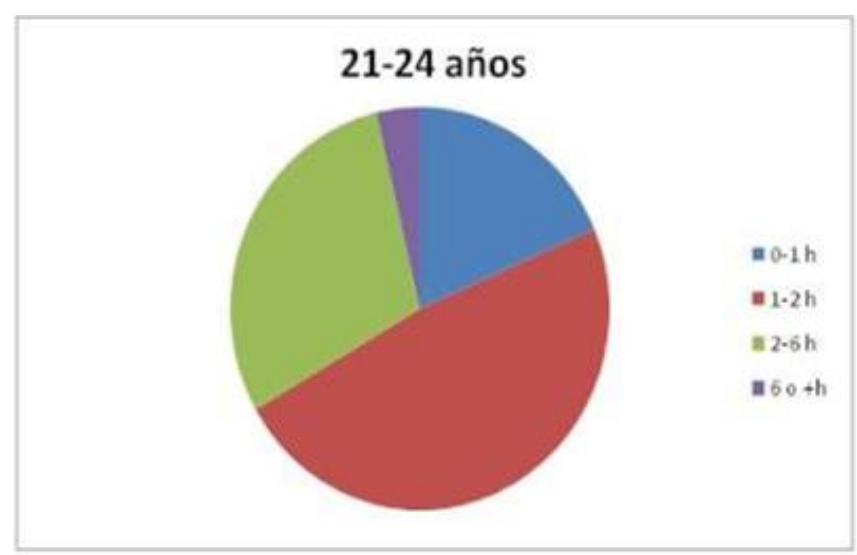


Las personas de 21 a 24 años encuestadas han dado similares conclusiones. Menos de la mitad han respondido que escuchan música entre 1 y 2 horas al día. La otra mitad ha respondido mayoritariamente entre 2 y 6 horas.

La opción más seguida es 0-1 horas, mientras que, igualmente que la anterior, un mínimo grupo de personas escuchan más de 6 horas al día. Luego del análisis de datos presentados por la autora, se concluye que los jóvenes entre 16 y 24 años escuchan música:

Acorde a sus estados de ánimo

- En lugares como:

$\checkmark$ la habitación

$\checkmark$ la calle (audífonos)

- $\quad$ El auto (> de 20 años)

- A cualquier hora del día

$\checkmark$ Móvil

$\checkmark$ Ipod

- De manera gratuita (youtube)

- Alguna vez han pagado

$\checkmark$ Conciertos

$\checkmark \mathrm{CD}$

- Prefieren la música Pop

$\checkmark$ seguida muy de cerca por el Rock

$\checkmark$ continuada por la Electrónica.

- La escuchan solos o acompañados

- Complementan otras actividades

La selección musical de los jóvenes de este rango de edad, depende del ritmo, letra y melodía, tomados como elementos de investigación; en ciertos casos la moda y en todos los casos determinados por su preferencia personal, lo cual puede variar con la etapa de madurez en que se encuentre.

En el último punto del análisis (9), se dice que complementan otras actividades; aquí se presenta la sustentación del tema a tratar. La música se puede utilizar libremente como herramienta de estudio porque cumple con todos los datos arriba señalados. El maestro puede servirse de ésta, como herramienta educativa para activar la clase y dejar que el alumno escoja la melodía de su preferencia como medio de análisis literario, profundizando en su letra, sentido, profundidad, rima, calidad literaria, etc. Además, podría darle alternativas, al trastocar la letra y cambiarla por conceptos científicos musicales, o al contrario, cambiar la música y tratar de incluir la letra, utilizando el pensamiento lógico creativo.

\section{¿Hasta dónde es prudente la libertad educativa?}

Es importante no perder el sentido lineal de la clase, y considerar que aunque la educación sea enfocada en la libertad, no puede perderse el hilo conductor porque carecería de propósito. El objetivo docente es cumplir con los objetivos planteados en los sílabos de las asignaturas, las 
técnicas y métodos son los que se modificarían, utilizando la herramienta musical para llegar a la literatura de las ciencias.

La calidad cultural de las letras es manejable a nivel académico, siempre y cuando los parámetros sean totalmente identificados, ya que puede tratarse la letra de una canción para detectar falencias y trabajar en la cultura crítica del alumno, esto le daría un amplio margen de análisis respecto a los trabajos musicales que presenta el entorno.

\section{El derecho a la Creatividad}

Tal como lo señala la Constitución de la República del Ecuador (Legales, 2016):

Art. 26.- La educación es un derecho de las personas a lo largo de su vida y un deber ineludible e in excusable del Estado. Constituye un área prioritaria de la política pública y de la inversión estatal, garantía de la igualdad e inclusió n social y condición indispensable para el buen vivir. Las personas, las familias y la sociedad tienen el derecho y la responsabilidad de participar en el proceso educativo.

Art. 27.- La educación se centrará en el ser humano y garantizará su desarrollo holístico, en el marc o del respeto a los derechos humanos, al medio ambiente sustentable y a la democracia; será participativa, obligatoria, intercultural, democ rática, incluyente y diversa, de calidad y calidez; impulsará la equidad de género, la justicia, la solidaridad y la paz; estimulará el sentido crí tico, el arte y la cultura física, la iniciativa individual y comunitaria, y el desarrollo de competencias y capacidades para crear y trabajar.

La educación es indispensable para el conocimiento, el ejercicio de los derechos y la const rucción de un país soberano, y constituye un eje estratégico para el desarrollo nacional. Amparados por la carta magna, los docentes e IES del Ecuador, deben (obligatoriedad) otorgar el derecho a que los alumnos se expresen con libertad y adopten las herramientas de aprendizaje que sean más adecuadas para ellos, tomando como referencia su criterio y desarrollo personal, acorde con los elementos que la cotidianidad les ofrece.

\section{Aplicación de estrategias}

Luego del análisis evidenciado en teorías y estudios de investigación tomando como herramienta la música, se considera que esta parte de la educación integral del alumno se debe manejar dentro del área de aspectos innovadores pedagógicos; los alumnos están en la capacidad de elegir dentro del repertorio musical la melodía que sea de su interés para aplicarla a las diferentes áreas de estudio.

En algunos casos se sentirán atraídos a utilizar la letra buscando la similitud de caracteres en las ciencias a estudiar; en otros, tomarán la música para fijar en su memoria el diseño teórico de un tema. Pedagógicamente, Jacques Dalcroze, sostiene que también se puede mantener una experiencia musical a nivel físico, por medio de movimientos que ordena las ideas de modo tal que se fijan en la memoria sucesos alusivos al tema.

Al parecer, la evolución educativa está reinventándose continuamente y no se puede dejar de lado el estudio de las herramientas a utilizarse, tomando en consideración los procesos de actualización pedagógica en donde se indica que el alumno no es un mero espectador, sino que se 
convierte en un protagonista de su educación, señalando los parámetros con los que desea aprehender y los medios más acordes a ese ritmo educativo.

En algunos casos, los docentes no se encuentran preparados para esta aceleración educativa, sin embargo, no se puede frenar el curso de su aprendizaje, por lo que es recomendable prodigar las herramientas adecuadas para dicho proceso, dejando que sea el alumno quien determine el modo, ritmo y circunstancia en que le es más propicio aprender y con esto cumplir con la función actual del docente, servir de facilitador en el proceso de enseñanza-aprendizaje.

\section{Bibliografía}

Arana, R. (22 de Noviembre de 2015). La República. Obtenido de http://larepublica.pe/impresa/enportada/720076-543-de-los-jovenes-prefieren-lamusica-de-los-anos-80

Artística", N. E. (2 de Abril de 2009). La retreta. Obtenido de <http://www.laretreta.net/0202/orff.pdf>

Bocaz Claudia Silva, Rocío campos. (2003). Metodo Maria Montessori. Obtenido de http://www.elviajerosuizo.com/resources/metodo.montessori-resumen.pdf:

http://www.elviajerosuizo.com/resources/metodo.montessori-resumen.pdf

Bressan, A. P., \& Bressan, O. (2008). Probabilidad y Estadística: Cómo trabajar con niños y jóvenes. Buenos Aires-Argentina: Novedades Educativas.

Buenaventura, E. (2009). El Método De Creación Colectiva En La Propuesta Didáctica Del Maestro Enrique Buenaventura: Anotaciones Obtenido de file:///C:/Users/Teresa/Downloads/DialnetElMetodoDeCreacionColectivaEnLaPropuestaDidactica D-4016517.pdf:

file:///C:/Users/Teresa/Downloads/DialnetElMetodoDeCreacionColectivaEnLaPropuestaDidactica D-4016517.pdf

Bull, M. (2010). iPod: un mundo sonoro personalizado para sus consumidores. Revista Científica de Educomunicación; ISSN: 1134-3478; páginas 55-63, 57, 58.

Carmen Gómez Granel,César Coll Salvador. (1994). De qué hablamos cuando hablamos de constructivismo.

Obtenido

http://w.paideia.synaptium.net/pub/pesegpatt2/metodologia/met_Constructiv.rtf:

http://w.paideia.synaptium.net/pub/pesegpatt2/metodologia/met_Constructiv.rtf

Clásicos, L. (2015). La República(Platón). Obtenido de http://www.acanomas.com/LibrosClasicos/8161/La-Republica-(Platon).htm Dalcroze, J. (1926).

Dalcroze Eurhythmics. Génevé: Institut Jacques Dalcroze.

Díaz, A. M. (2015). Asignaturas de la Mención en Educación Musical. Obtenido de Preferencias musicales de los jovenes: https://sites.google.com/site/mencionmusical3/home/musica-ycultura/preferenciasmusicales-de-los-jovenes

Educacìon, M. d. (2016). Acuerdo Nro. MINEDUC-ME-2016-00020-A . Acuerdo Nro. MINEDUC-ME2016-00020-A . Quito, Pichincha, Ecuador: Quipux. 
Educacion, M. d. (2016). Ministerio de Educacion . Recuperado el 21 de 07 de 2016, de http://educacion.gob.ec/actualizacion-curricular/ Educarm. (2010). La Filosofía de Platón. Obtenido de https://servicios.educarm.es/templates/portal/images/ficheros/.../9/.../tema_platn.doc

Educarm. (s.f.). La filosofía de Platón. Obtenido de La filosofía de Platón: https://servicios.educarm.es/templates/portal/images/ficheros/.../9/.../tema_platn.doc

García, J. A.-Q. (Mayo de 2000). Investigación Cualitativa en Educación Musical: un nuevo reto en el contexto educativo español. Obtenido de http://musica.rediris.es/leeme/revista/rodriguezja00.pdf

Gardner, H. (1983). Inteligencias Multiples, la teorìa en la pràctica. Cambridge: New Horizons.

Goleman, D. (1996). Inteligencia Emocional. New York: kaìros.

Legales, G. d. (2016). Constitución De La República Del Ecuador. Obtenido De Sección V. Educación. Art. 26 y 27: http://www.pucesi.edu.ec/web/wpcontent/uploads/2016/04/Constituci\%C3\%B3n-dela-Republica-2008..pdf

Ministerio de Educación, C. y. (2001). La Educación Artística clave para el desarrollo de la Creatividad. Madrid: Secretaría General Técnica. Fareso, S.A.

Núñez, J. C. (2009). Motivación, Aprendizaje Y Rendimiento Académico. Obtenido de http://www.educacion.udc.es/grupos/gipdae/documentos/congreso/xcongreso/pdfs/cc/ cc3.pdf: http://www.educacion.udc.es/grupos/gipdae/documentos/congreso/xcongreso/pdfs/cc/ cc3.pdf

Ortí, A. S. (2013). Indicadores Básicos de Juventud. Obtenido de Centro Reina Sofía de Madrid: http://www.ciong.org/doc/general/indicadores-basicos-juventud.pdf

Regade, B. (2012). Psicología y Mente. Obtenido de https://psicologiaymente.net/desarrollo/teoriadesarrollo-lenguaje-noam-chomsky\#

Soler, E. A. (2002). Bases Lingüísticas y Metodológicas para la enseñanza de la Lengua Inglesa. Castelló de la Plana: Publicaciones de la Universitat Jaume I. Servei de Comunicació i Publicacions.

Swanwick, K. (2000). Music, Mind and Education. Madrid: Ediciones Morata, S.L.

Swanwick, K. (2000). Música, Pensamiento y Educación. Obtenido de https://books.google.com.ec/books?id=TBycYcIN2XoC\&printsec=frontcover\&dq=es trategias+metodologicas+educar+en+libertad+musica\&hl=es419\&sa=X\&redir_esc=y\#v=onepage $\& q \& f=$ false Unesco. $(1991)$. portal

Unesco org. Recuperado el 21 de 07 de 2016 de http://portal.unesco.org/es/ev.phpURL_ID=26463\&URL_DO=DO_TOPIC\&URL_SECTION=201. html

Vernia, A. M. (2012). Método Pedagógico Musical Dalcroze. Obtenido de https://dialnet.unirioja.es/descarga/articulo/3946014.pdf 